\title{
Clinical Applications of the Thoracodorsal Artery Perforator Flap in Extremity Soft Tissue Reconstruction
}

\author{
SHERIF A. HANTASH, M.D.*; WAEL M. AYAD, M.D.**; AMGAD A. HENDY, M.D.* and \\ MOHAMED S. SADAKA, M.D.* \\ The Department of Plastic and Reconstructive Surgery, Faculties of Medicine, Tanta* and Al-Azhar** Universities
}

\begin{abstract}
Introduction: For a long time, the free or pedicled thoracodorsal artery perforator (TDAP) flaps were used for reconstruction of large cutaneous defects. These may be regional soft tissue defects of trunk, axilla and breast where the pedicled flap used, or distant tissue defects of face, elbow, forearm and lower extremity where the free TDAP flap is used.
\end{abstract}

Aim of the Study: This study aimed to evaluate the clinical applications of the thoracodorsal artery perforator flap (TDAP) either free or pedicled in the reconstruction of soft tissue defects of extremities.

Patients and Methods: This study was conducted on 20 patients admitted to the Plastic Surgery Departments of both Tanta \& Al Azhar Universities during the period from September 2015 to December 2017. These patients were presented by extremity soft tissue defects that required a flap reconstruction.

Results: The results showed that the TDAP flap is a versatile and robust flap in soft tissue reconstruction of the extremities. This flap has great advantages as a long vascular pedicle; large skin paddle can be harvested, minimal donor site morbidity and thin skin paddle to resurface shallow defects. The TDAP flap showed also some drawbacks like meticulous and time-consuming dissection of the perforators, donor site scar widening.

Conclusion: TDAP flap is a versatile and an excellent flap either free or pedicled for extremity soft tissue reconstruction. It should be added to the workhorse perforator flaps.

Key Words: Thoracodorsal - Flap - Perforator - Extremity.

\section{INTRODUCTION}

Although Latissimus dorsi (LD) musculocutaneous flap is still widely used, either pedicled or as free transfers, commonly encountered restrictions are bulkiness, loss of muscle function, long-lasting seroma formation, and contour deformities at the donor site [1]. These factors, in addition to developing microsurgical skills and expanding anatomic knowledge in flap surgery, lead to refinements in recipient aesthetics and minimizing donor site morbidity. Perforator flaps became the most attractive option among many reconstructive surgeons [2].

Pedicled TDAP flap was used for axillary and breast defects, and with cadaveric dissections it was shown that the arc of rotation allows shoulder, chest wall, neck, and proximal arm reconstruction in the same fashion [3-5]. Free transfer of the flap for shallow defects on different body regions was also reported in some other clinical series [6]. Even in the pediatric population, the use of this flap is safe due to its relatively constant vascular anatomy [7].

\section{Aim of the study:}

The aim of this study is to evaluate the clinical applications of thoracodorsal artery perforator flap (TDAP) either free or pedicled in reconstruction of soft tissue defects of extremities.

\section{PATIENTS AND METHODS}

This is a prospective study that was performed on 20 patients. Patients were admitted and operated in Departments of Plastic and Reconstructive Surgery, Faculty of Medicine, Tanta and Al-Azhar Universities during the period from September 2015 to December 2017.

All patients included in this study were presented by extremity soft tissue defects resulting from either trauma, excision of skin malignancies, chronic ulcers or release of wide scar contractures. These defects were not feasible for primary closure or reconstruction using skin grafting. Patients excluded from the study are those not fit for surgery, suffering 
from extremity vascular disease, subjected to previous LD flap harvest or axillary dissection.

All patients were operated under general anesthesia; a broad-spectrum antibiotic was given intravenously with anesthesia induction. Informed consent was obtained from all cases and all cases were subjected to thorough clinical assessment, routine preoperative investigations and Duplex ultrasonography or computerized tomographic angiography.

\section{Preoperative marking: (Fig. 1A)}

The donor site was ipsilateral in all pedicled flap cases and contralateral in all free flap cases. The anatomical landmarks included were: Outline of the scapular angle, posterior axillary fold and free or lateral border of LD muscle represented by a line from posterior axillary fold to posterior superior iliac spine.

A handheld Doppler ( $8 \mathrm{MHz}$ ) was used to localize and mark thoracodorsal artery (TDA) perforators on the skin of back. For perforators derived from descending branch of TDA, a circle of a 3$\mathrm{cm}$-radius centered at a point $2 \mathrm{~cm}$ medial to lateral border of latissimus dorsi muscle at the level of inferior scapular angle is drawn. For perforators derived from transverse branch of TDA, a 3-cmradius circle centered at the inferior scapular angle is drawn [8].

The TDAP flap was raised as a fasciocutaneous flap based on the perforators of TDA with sparing of LD muscle and TD nerve. Flap dimensions varied according to defect. The perforator does not need to be centered to reliably perfuse this flap as eccentric position of the perforator in the flap is quite safe and ensures a longer pedicle [9].

We used two designs for the skin paddle: Vertical skin paddle (along lateral border of LD muscle based on perforators from descending branch of TDA) and horizontal skin paddle (based on perforators of descending and transverse branches).

\section{Operative technique: (Fig. 1B-F)}

With the aid of a magnifying loupe (3.5 X), the antero-inferior part of the flap is incised, dissecting through the superficial fascia overlying the LD muscle using a monopolar diathermy in search for any direct or septocutaneous perforator. Once a suitable perforator is identified, it is followed up to the point where it exits the LD muscle and followed to TDA to confirm its origin. A suitable perforator is an artery accompanied by two venae comitantes and shown to be pulsating intraoperative. Then dissection is continued using scissors to avoid vessel spasm. Once the lateral border of LD muscle is identified, the anterosuperior part of incision is made.

At this point, if flap is planned to be sensate, the posterior cutaneous branches of the lateral intercostal nerves are preserved. The thoracodorsal pedicle is dissected until sufficient length and adequate caliber is gained. If extra length is needed, the transverse branch of TDA is ligated or clipped and dissection can continue up to the origin of circumflex scapular vessels from the subscapular trunk. After complete detachment of skin paddle from muscle and ligation of the descending branch of TDA distal to the perforator, good capillary filling is confirmed. A second team works simultaneously for recipient site preparation which once complete the TD pedicle divided and flap ready for transfer.

The microsurgical anastomosis was done using a magnifying loupe $(4.5 \mathrm{X})$ and microsuture (polypropylene 9/0) with arterial one first in an end to end fashion and a bolus dose of heparin 5000 I.U was given intravenously by the anesthesiologist who was instructed to keep the patient well hydrated and avoid hypotension and vasopressors. The operating microscope was not used in any case, even in the pediatric case. The anastomosis is checked for patency; the skin paddle is checked also for confirmation of adequate perfusion. Complete inset of the flap is done by suturing the borders of the skin paddle to the edges of defect without tension. A Penrose drain is inserted under the skin paddle. Donor site was close primarily in 2 layers (skin and subcutaneous tissue) if width less than $10 \mathrm{~cm}$, otherwise a split-thickness skin graft was used.

Postoperative care: Included adequate positioning to avoid pressure on the flap, activity restriction, limb elevation, warming, hydration, follow-up of vital data, twice daily dose of low molecular weight heparin, broad spectrum antibiotics and pain killers. Monitoring of the flap was started immediately post-operative and repeated every 30-60 minutes for the first 24 hours, every hour on the second and third postoperative days, and four times daily thereafter until the patient is discharged. The monitoring was based on clinical examination of the flap including the color, temperature, turgor and capillary refill and assisted with handheld Doppler. 

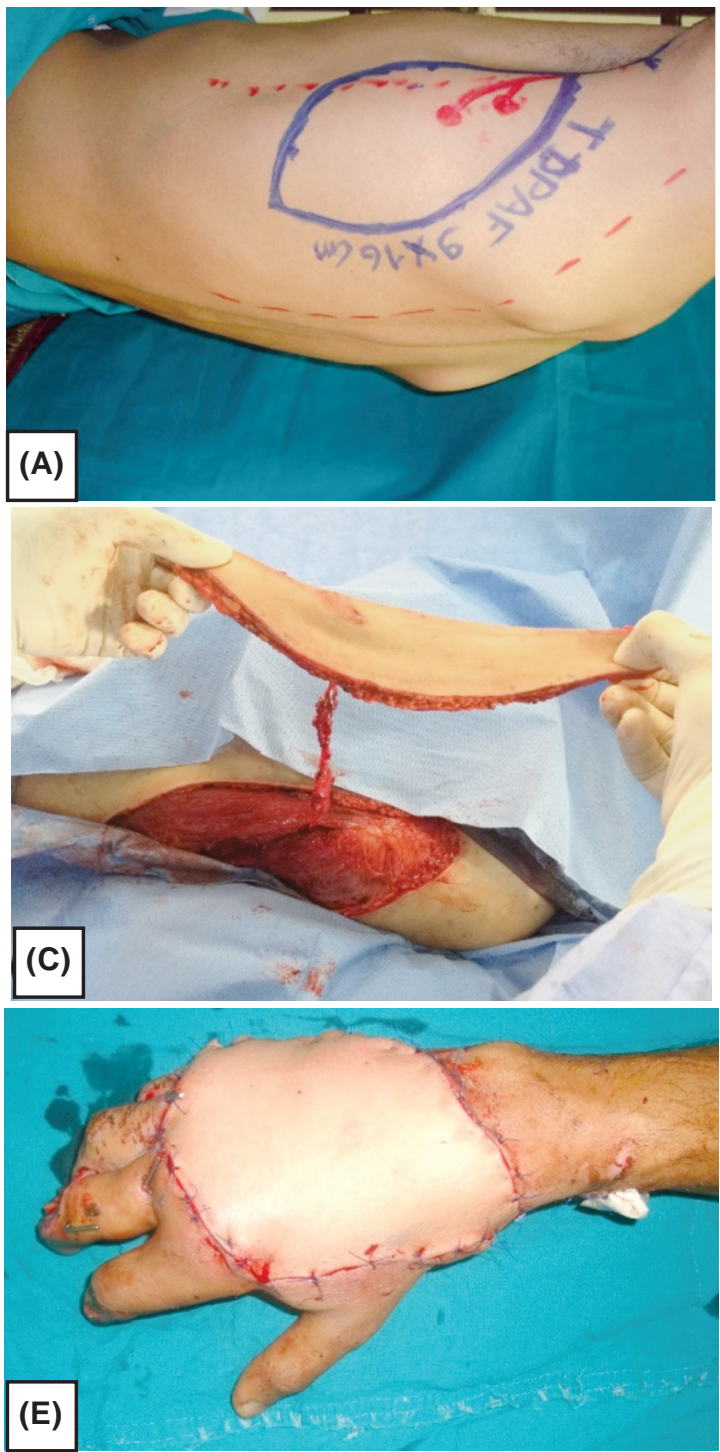
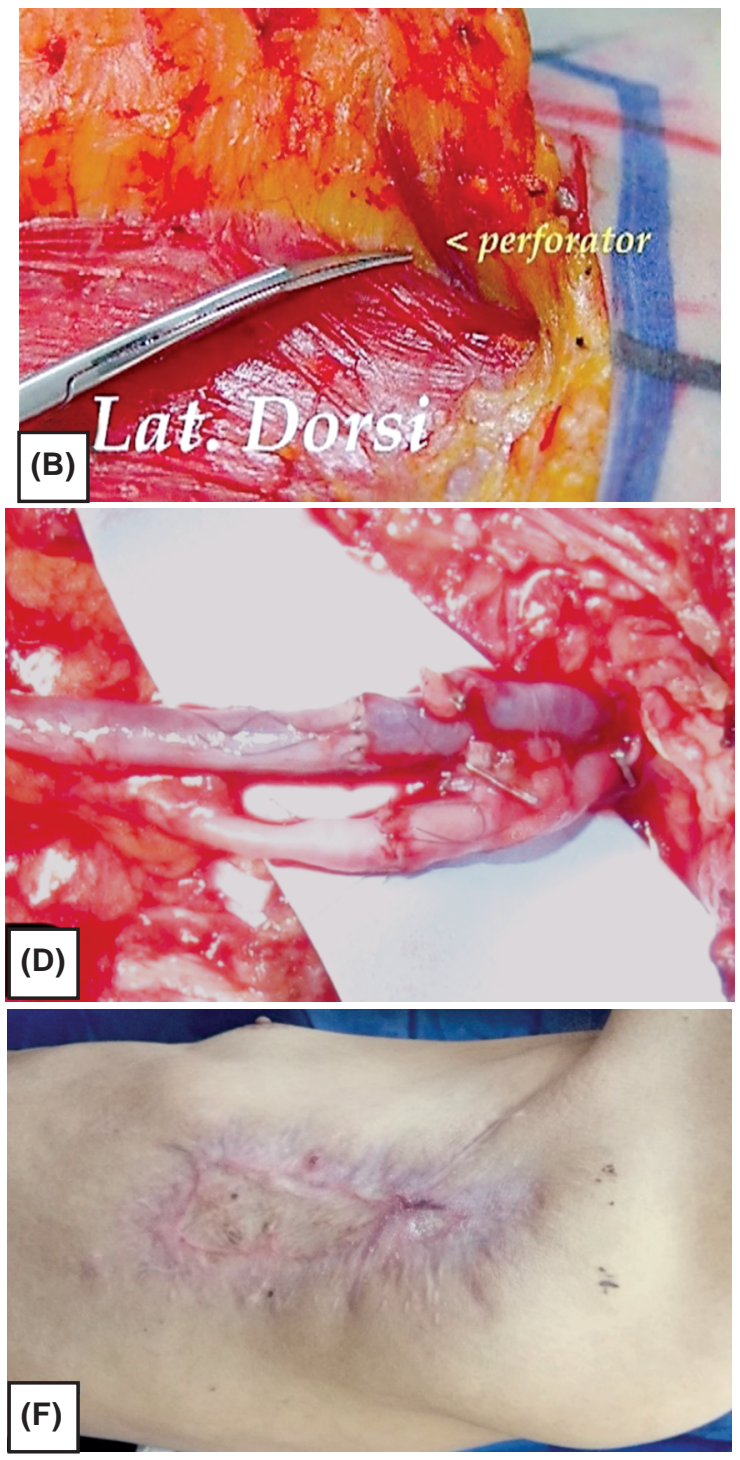

(F)

Fig. (1): (A): Vertical design of TDAP flap and marked perforators. (B): TDA perforator. (C): Complete freeing of the flap, the skin paddle is only attached by the pedicle. (D): Microsurgical anastomosis of TDA and vein to recipient vessels. (E): The skin paddle after complete inset. (F): Split thickness skin grafting of the TDAP flap donor site.

\section{RESULTS}

Figs. (2,3):

This prospective study included 20 patients that were presented with extremity soft tissue defects requiring flap reconstruction during the period from September 2015 to December 2017. The patient ages ranged from 9 to 55 years (mean age: $31.8 \pm 13.45 \mathrm{SD}$ ). Five females $(25 \%)$ and 15 males $(75 \%)$ were included.

The most common cause of soft tissue defects was post traumatic ( 12 cases, 60\%, 10 males \& 2 females) followed by release after post burn contractures ( 4 cases, 20\%, 2 males \& 2 females), then after excision of skin malignancies ( 3 cases, $15 \%$, 2 males \& 1 female) and excision of unstable scar ( 1 case, $5 \%, 1$ male). Thirteen cases were presented by soft tissue defects in the lower limb (65\%) and 7 cases were in the upper limb and axilla (35\%), 4 cases were reconstructed by pedicled flaps $(20 \%)$ and the remaining 16 cases with free flaps $(80 \%)$. The mean operative time was 390 minutes (range 300-540 minutes) for the free flaps, and 255 minutes (range 210-300 minutes) for the pedicled flaps. Most of the time was spent in the intramuscular dissection of perforators where the mean time was 183 minutes (range 150-240 minutes).

The flap dimensions ranged from $12 \times 7 \mathrm{~cm}$ $\left(84 \mathrm{~cm}^{2}\right)$ to $26 \mathrm{x} 11 \mathrm{~cm}\left(286 \mathrm{~cm}^{2}\right)$. All flaps were based on a single perforator. The thoracodorsal pedicle lengths ranged from 14 to $23 \mathrm{~cm}$ (mean 16.8). The donor site was closed primarily in 16 cases $(80 \%$, flap widths $\leq 10 \mathrm{~cm})$ while it was skin grafted in 4 cases $(20 \%$, flap widths $>10 \mathrm{~cm})$. 

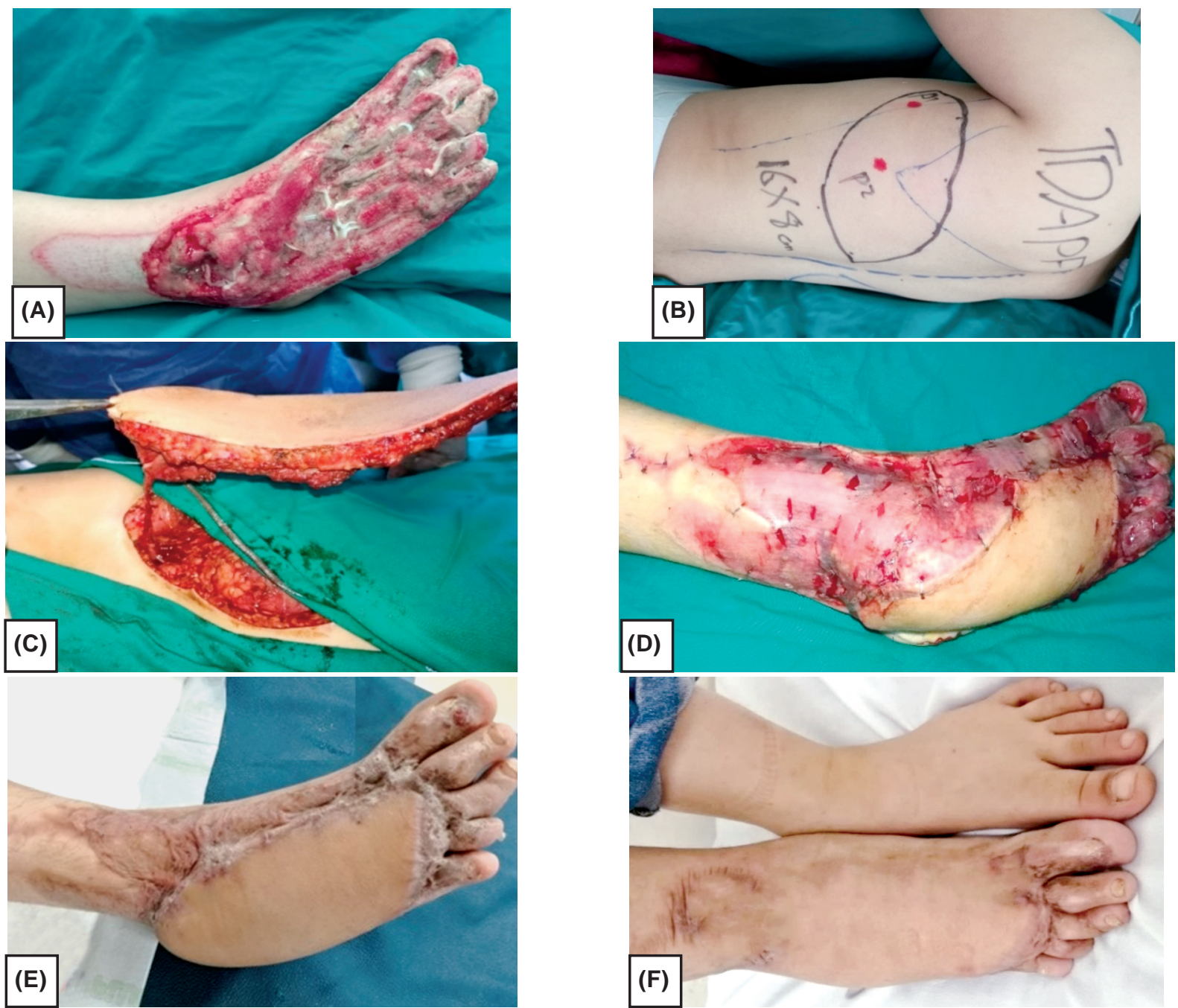

Fig. (2): (A): Crushed right foot after debridement of the wound. (B): Marking of transverse TDAP flap skin paddle. (C): Flap after isolation of the skin paddle with muscle cuff harvested around the pedicle. (D): Flap after inset over exposed bone, other parts of the defect covered by STSG. (E): Flap 3 months postoperative. (F): Flap after debulking.
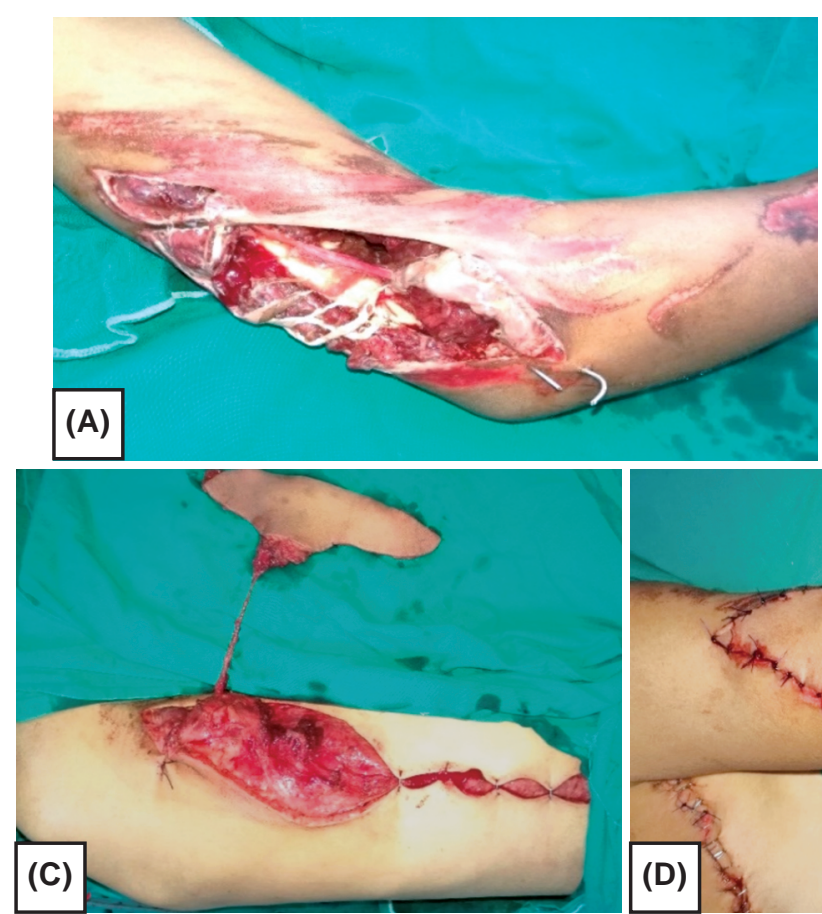

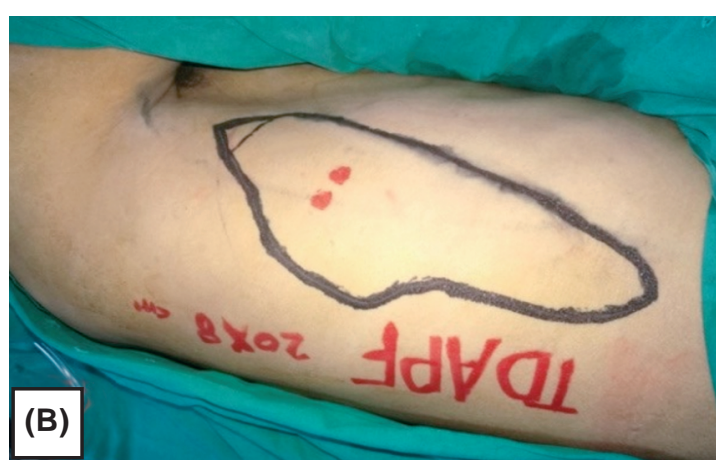

Fig. (3): (A): Crushing trauma of the Rt. arm with soft tissue loss and exposed humerus and radial nerve. (B): Pedicled TDAP flap was fashioned $(20 \times 8 \mathrm{~cm})$. Distal perforators from the descending branch of the TDA were localized. (C): Harvesting of pedicled TDAP flap. The skin paddle is attached by the TD pedicle with muscle cuff included. (D): The flap was inset through a subcutaneous tunnel. 
The outcome of TDAP flap in our study was assessed as follows:

A- The aesthetic outcome of TDAP flap was assessed using the Likert scale [10], in which four main factors are evaluated on a numerical scale: General appearance, Contour, Color and texture and the score is classified as follows: 4 to 6 ; poor, 7 to 9; bad, 10 to 13 ; regular, 14 to 16 ; good and 17 to 20 ; very good.

This evaluation was performed for each case in this study by three plastic and reconstructive surgeons and the patient. Each one of the four evaluated aesthetic aspects was analyzed. These factors were compared with the features of a normal extremity on a scale of 1 to 5 in which 1 was the worst rating and 5 was the best as follows: Strongly disagree, disagree, neither agree nor disagree, agree (Table 1). Evaluation of general aesthetics revealed a mean of 13.5 (regular) with the value of each characteristic evaluated as shown in Fig. (4).

\section{B- Functional outcome:}

1- Upper extremity: Was evaluated by the QuickDASH scale [11] which assesses motor functions of the upper extremity using a simple questionnaire of 11 items which was answered by the patients 3-6 months post-operative. This study included 7 cases of upper extremity defects reconstructed by 4 free and 3 pedicled TDAP flaps, the functional outcome as evaluated by the QUICKDASH score was $(22.6 \%)$ as a mean value (Fig. 5).

2- Lower extremity: Was assessed by using the lower limb core scale (LLCS) [12]. This questionnaire was answered by the patients 3-6 months post-operative.

\section{Post-operative complications:}

1- TDAP flap complications occurred in 9 cases (45\%) as follows:

- Three cases of total flap loss (15\%), all were in the lower extremity, 2 of them were due to anastomosis done within the zone of injury with post traumatic vessel disease. Although 2 revisions were done for the arterial anastomosis to salvage the 2 flaps, unfortunately they didn't pass. The third case of total flap loss was due to arterial compromise secondary to arterial thrombus found during exploration of the flap because of flap ischemia. Thrombectomy and revision of arterial anastomosis were done but unfortunately, the flap did not pass.

- Four cases of post-operative flap venous congestion $(20 \%)$ ( 3 were in lower limb, one in upper limb), 2 of them were managed conserva- tively in the form of limb elevation, full anticoagulation and removal of some sutures fixing the skin paddle and the flaps went well. The other 2 flaps required intervention and exploration where one of them showed a venous thrombus and was managed by thrombectomy and revision of venous anastomosis (this flap ended by partial loss. The other one showed a hematoma under the flap and was managed by drainage and a negative pressure wound therapy was applied to one side of the skin paddle after removal of skin sutures, this flap went well after that.

- Two cases of wound infection (10\%), one of them was managed conservatively by wound swab, culture \& sensitivity and proper antibiotics with daily wound care. The other case showed a wound dehiscence that required re-suturing after wound care.

\section{2- Post-operative donor site complications:}

They occurred in 5 cases (25\%), 3 cases of wound dehiscence ( 2 of them were simple and managed conservatively by local wound care, the $3^{\text {rd }}$ one required revision of the wound), one case of wound infection that was managed by local wound care and one case of seroma that was managed by repeated aspiration.

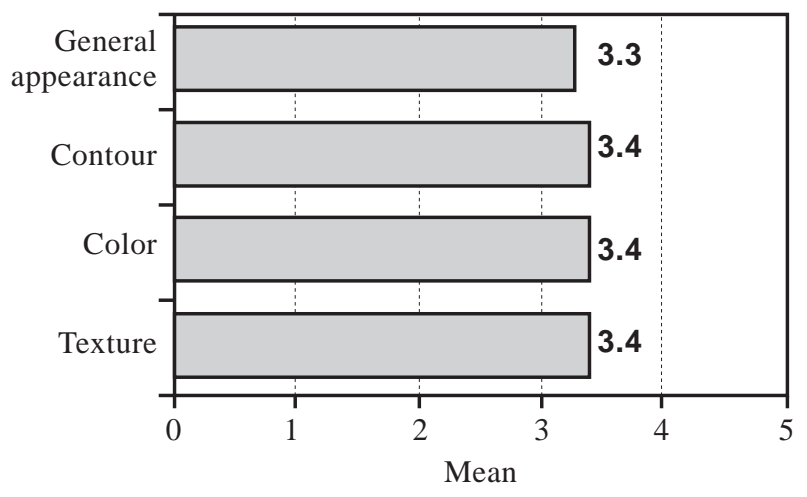

Fig. (4): Aesthetic evaluation characteristics.

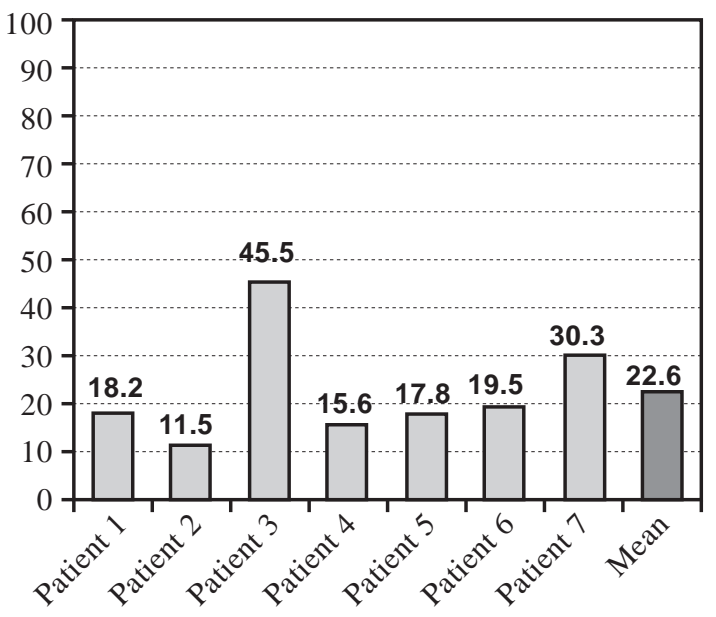

Fig. (5): Rating of the QUICKDASH score. 


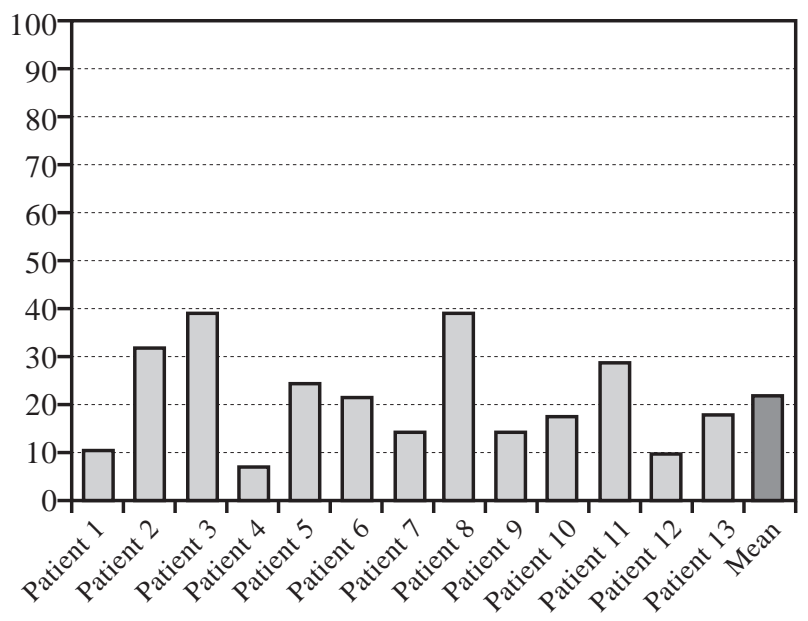

Fig. (6): Functional outcome of the TDAP flaps used in lower extremity reconstruction evaluated by the lower limb core scale (LLCS).

Table (1): Post-operative aesthetic outcome according to Likert scale.

\begin{tabular}{llll}
\hline Case & Min/Max & Mean & Qualification \\
\hline$(1)$ & $15 / 18$ & 16.5 & Good \\
$(2)$ & $10 / 13$ & 11.5 & Regular \\
$(3)$ & $14 / 17$ & 15.5 & Good \\
$(4)$ & $4 / 4$ & 4 & Poor \\
$(5)$ & $17 / 19$ & 17.8 & Very good \\
$(6)$ & $13 / 16$ & 14.5 & Good \\
$(7)$ & $16 / 19$ & 17.5 & Very good \\
$(8)$ & $10 / 12$ & 10.8 & Regular \\
$(9)$ & $10 / 12$ & 11 & Regular \\
$(10)$ & $14 / 16$ & 15.3 & Good \\
$(11)$ & $16 / 18$ & 17 & Very good \\
$(12)$ & $11 / 12$ & 11.5 & Regular \\
$(13)$ & $15 / 16$ & 15.5 & Good \\
$(14)$ & $10 / 12$ & 11.3 & Regular \\
$(15)$ & $14 / 16$ & 14.8 & Good \\
$(16)$ & $7 / 9$ & 8 & Bad \\
$(17)$ & $14 / 16$ & 15 & Good \\
$(18)$ & $14 / 16$ & 14.8 & Good \\
$(19)$ & $11 / 13$ & 11.8 & Regular \\
$(20)$ & $16 / 18$ & 17 & Very good \\
\hline
\end{tabular}

\section{DISCUSSION}

Although the LD musculocutaneous flap is one of the most reliable flaps in reconstructive surgery, the bulk of the flap and the donor site morbidities change the direction towards harvesting a perforator flap and preserve the muscle function. In addition, the denervated muscle included in the flap undergoes unpredictable degree of atrophy, making the volume of reconstruction more doubtful [13].

The ages of patients in our study ranged from 9 to 55 years, the only pediatric case was 9 years old. The most common cause of soft tissue defects in this study was post-traumatic $(60 \%)$ and that explains why males are more involved in this study $(75 \%)$ as males are more subjected to trauma.
The flap dimensions were adjusted according to the defect dimensions and this was judged after preparation of the recipient site. The donor site was closed primarily in 16 cases $(80 \%)$. Although there are some studies denoting the ability to primarily close the donor site of the TDAP flap up to $12 \mathrm{~cm}$ width [14], we found that the maximum width that can be closed primarily is $10 \mathrm{~cm}$ and this is attributed to the presence of all intact LD muscle in the field.

After analyzing results of Likert scale [99] we found that the cases where a split thickness skin graft was applied beside the flap either to cover the pedicle or to cover a graftable area not covered by the flap have a low score regarding the general appearance evaluated by the patients, this is attributed to patients evaluating the whole area of reconstruction not the flap alone.

The mean value of QUICKDASH score in this study [11] was (22.6\%) which represents a low disability that means that most of the common functions of the upper limb, as grabbing or taking a pen or a glass of water, could be done. The lower limb core scale (LLCS) post-operative mean value was 21.5 indicating that the patients after 3 months can perform some functions related to lower limb with mild degree like going up and down stairs, walking on flat surfaces and putting on or taking off socks or stockings.

The donor site complications occurred in 5 cases $(25 \%)$. The seroma formation rates with LD musculocutaneous flap are reportedly as high as $5 \%$ to $80 \%[15,16]$. Seroma formation may increase the risk of wound dehiscence and secondary infection requiring serial needle aspirations. The sacrifice of the muscle together with the lumbar fat used in extended flaps are the main causes of post-operative seroma formed after LD myocutaneous flaps [17]. The TDAP flap was reported to preserve LD muscle reducing the postoperative drainage and risk of seroma formation $[\mathbf{1 4 , 1 7 , 1 8 , 2 1 ]}$. This correlates with our study (only 1 case of post-operative seroma, $5 \%$ ). This will lead to shorter hospital stay, less frequent hospital visits, and lower medical cost.

Many studies have evaluated the TDAP flap in extremity soft tissue reconstruction where they reported that this flap has many advantages: Comparable thickness to that of extremities, allow two team approach, lower incidence of pedicle affection by atherosclerotic changes, suitable pedicle length (up to $23 \mathrm{~cm}$ ) and diameter (up to $2 \mathrm{~mm}$ ), low donor site morbidity, stable coverage, accepted aesthetic appearance, primary closure of donor site with flap width up to $10 \mathrm{~cm}$, donor scar can be hidden by 
underwear, possibility of sensate flap, possible chimeric or combined flap including muscle or bone, versatility of skin paddle orientation and defatting leaving $2 \mathrm{~cm}$ subcutaneous tissue to cover shallow defects $[\mathbf{1 3 , 2 1 , 2 2 ]}$.

They also reported some disadvantages of the TDAP flap as increased operative time by patient positioning and perforator dissection, possible donor site scar widening and hypertrophy, skin paddle still bulky in obese patients and the need for meticulous preoperative planning and perforator mapping $[\mathbf{1 4 , 2 3}]$.

\section{Conclusion:}

The TDAP flap is a versatile alternative in extremity soft tissue reconstruction both as a free and as a pedicled flap, which may be used for a wide range of indications in appropriately selected patients. This flap should be one of the workhorse flaps in extremity soft tissue reconstruction owing to its great advantages and versatility. The drawbacks of the TDAP flap can be manipulated by some technical modifications.

\section{REFERENCES}

1- Russell R.C., Pribaz J., Zook E.G., Leighton W.D., Eriksson E. and Smith C.J.: Functional evaluation of latissimus dorsi donor site. Plast. Reconstr. Surg., 78: 336, 1986.

2- Titley O., Spyrou G. and Fatah M.: Preventing seroma in the latissimus dorsi flap donor site. Br. J. Plast. Surg., 50: 106, 1997.

3- Guerra A.B., Metzinger S.E., Lund K.M., Cooper M.M., Allen R.J. and Dupin C.L.: The thoracodorsal artery perforator flap: Clinical experience and anatomic study with emphasis on harvest techniques. Plast. Reconstr. Surg., 114: 32, 2004.

4- Angrigiani C., Rancati A., Escudero E. and Artero G.: Extended thoracodorsal artery perforator flap for breast reconstruction. Gland Surg., 4: 519, 2015.

5- Thomsen J.B. and Gunnarsson G.L.: The evolving breast reconstruction: From latissimus dorsi musculocutaneous flap to a propeller thoracodorsal fasciocutaneous flap. Gland Surg., 3: 151, 2014.

6- Heitmann C., Guerra A., Metzinger S.W., Levin L.S. and Allen R.J.: The thoracodorsal artery perforator flap: Anatomic basis and clinical application. Ann. Plast. Surg., 51: 23, 2003.

7- Kim S.E., Rhyou I.H., Suh B.G. and Chung K.C.: Use of thoracodorsal artery perforator flap for soft tissue reconstruction in children. Ann. Plast. Surg., 56: 451, 2006.

8- Mun G.H., Kim H.J., Cha M.K. and Kim W.Y.: Impact of perforator mapping using multidetector-row computed tomographic angiography on free thoracodorsal artery perforator flap transfer. Plast. Reconstr. Surg., 122: 1079, 2008.

9- Kim Y.H., Lee H.E., Lee J.H., Kim J.T. and Kim S.W.: Reliability of eccentric position of the pedicle instead of central position in a thoracodorsal artery perforator flap. Microsurgery, 37: 44, 2017.

10- Parrett B.M., Bou-Merhi J.S., Buntic R.F., Safa B., Buncke G.M. and Brooks D.: Refining outcomes in dorsal hand coverage: consideration of aesthetics and donor-site morbidity. Plast. Reconstr. Surg., 126: 1630, 2010.

11- Angst F., Schwyzer H.K., Aeschlimann A., Simmen B.R. and Goldhahn J.: Measures of adult shoulder function: disabilities of the arm, shoulder, and hand questionnaire (DASH) and its short version (QuickDASH), shoulder pain and disability index (SPADI), American Shoulder and Elbow Surgeons (ASES) Society standardized shoulder assessment form, Constant (Murley) score (CS), simple shoulder test (SST), Oxford shoulder score (OSS), shoulder disability questionnaire (SDQ), and Western Ontario shoulder instability index (WOSI). Arthritis Care Res., 63: S174, 2011.

12- Monticelli A., Ciclamini D., Boffano M., Boux E., Titolo P. and Panero B.: Lower limb core scale: A new application to evaluate and compare the outcomes of bone and softtissue tumours resection and reconstruction. Biomed Res. Int., 2014: 652141, 2014.

13- Chen S., Chen T. and Wang H.: Free thoracodorsal artery perforator flap in extremity reconstruction: 12 cases. Br. J. Plast. Surg., 57: 525, 2004.

14- Sever C., Uygur F., Kulahci Y., Karagoz H. and Sahin C.: Thoracodorsal artery perforator fasciocutaneous flap: A versatile alternative for coverage of various soft tissue defects. Indian J Plast. Surg., 45: 478, 2012.

15- Bailey S.H., Oni G., Guevara R., Wong C. and Saint-Cyr M.: Latissimus dorsi donor-site morbidity: The combination of quilting and fibrin sealant reduce length of drain placement and seroma rate. Ann. Plast. Surg., 68: 555, 2012.

16- Gruber S., Whitworth A.B., Kemmler G. and Papp C.: New risk factors for donor site seroma formation after latissimus dorsi flap breast reconstruction: 10-year period outcome analysis. J. Plast. Reconstr. Aesthet. Surg., 64: 69, 2011.

17- Arikawa M., Miyamoto S., Fujiki M., Higashino T., Oshima A. and Sakuraba M.: Comparison of Donor Site Drainage Duration and Seroma Rate Between Latissimus Dorsi Musculocutaneous Flaps and Thoracodorsal Artery Perforator Flaps. Ann. Plast. Surg., 79: 183, 2017.

18- Lee K.T., Kim A. and Mun G.H.: Comprehensive Analysis of Donor-Site Morbidity following Free Thoracodorsal Artery Perforator Flap Harvest. Plast. Reconstr. Surg., 138: 899, 2016

21- Jain L., Kumta S.M., Purohit S.K. and Raut R.: Thoracodorsal artery perforator flap; indeed a versatile flap. Indian J. Plast. Surg., 48: 153, 2015.

22- Kim K.N., Hong J.P., Park C.R. and Yoon C.S.: Modification of the Elevation Plane and Defatting Technique to Create a Thin Thoracodorsal Artery Perforator Flap. J. Reconstr. Microsurg., 32: 142, 2016.

23- Kulahci Y., Sever C., Uygur F., Oksuz S., Sahin C. and Duman H.: Pre-expanded pedicled thoracodorsal artery perforator flap for postburn axillary contracture reconstruction. Microsurgery, 31: 26, 2011. 